\title{
ClusterRR: A Record and Replay Framework for Virtual Machine Cluster
}

\author{
Wei Wang \\ Institute of Information Engineering, \\ School of Cyber Security, University \\ of Chinese Academy of Sciences \\ Beijing, China \\ wangwei2@iie.ac.cn
}

\author{
Zhiyu $\mathrm{Hao}^{*}$ \\ Institute of Information Engineering, \\ Chinese Academy of Sciences \\ Beijing, China \\ haozhiyu@iie.ac.cn
}

\author{
Lei Cui \\ Institute of Information Engineering, \\ Chinese Academy of Sciences \\ Beijing, China \\ cuilei@iie.ac.cn
}

\begin{abstract}
The Record and Replay (RnR) technology provides the ability to reproduce past execution of systems deterministically. It has many prominent applications, including fault tolerance, security analysis, and failure diagnosis. In system virtualization, previous RnR researches mainly focus on individual VM, including coherent replaying of multi-core systems, reducing performance penalty and storage overhead. However, with the emerging of distributed systems deployed in virtual machine clusters (VMC), the existing RnR technology of individual VM can not meet the requirements of analyzers and developers. The critical challenge for $\mathrm{VMC} \mathrm{RnR}$ is to maintain the consistency of global state. In this paper, we propose ClusterRR, a RnR framework for VMC. To solve the inconsistency problem, we propose coordination protocols to schedule the record and replay process of VMs. Meanwhile, we employ a Hybrid RnR approach to reduce the performance penalty and storage costs caused by recording network events. Moreover, we implement ClusterRR on QEMU/KVM platform and utilize a network packets retransmission framework to guarantee the reproducibility of VMC replay. Last, we conduct a series of experiments to measure its efficiency and overhead. The results show that ClusterRR would efficiently replay the execution of the whole VMC at instruction-level granularity.

CCS Concepts: - Software and its engineering $\rightarrow$ Operating systems; • Computer systems organization $\rightarrow$ Cloud computing.
\end{abstract}

Keywords: vitual machine cluster, deterministic replay, global consistency, non-deterministic event

${ }^{\star}$ Zhiyu Hao is the corresponding author.

This work is licensed under a Creative Commons Attribution 4.0 International License.

VEE '22, March 1, 2022, Virtual, Switzerland

( 2022 Copyright held by the owner/author(s).

ACM ISBN 978-1-4503-9251-8/22/03.

https://doi.org/10.1145/3516807.3516819
ACM Reference Format:

Wei Wang, Zhiyu Hao, and Lei Cui. 2022. ClusterRR: A Record and Replay Framework for Virtual Machine Cluster. In Proceedings of the 18th ACM SIGPLAN/SIGOPS International Conference on Virtual Execution Environments (VEE '22), March 1, 2022, Virtual, Switzerland. ACM, New York, NY, USA, 14 pages. https://doi.org/10.1145/ 3516807.3516819

\section{Introduction}

The Record and Replay ( $\mathrm{RnR}$ ) technology enables reproducing the system's previous execution by logging related information during the record stage. Due to the advantage of deterministic replay, it is applied to a wide variety of scenarios. For example, the RnR technology is used for system recovery by rolling back and replaying the execution [8]. In addition, for security analysis, it decouples detailed analysis from original execution [29, 34]. Moreover, software developers leverage RnR to reproduce non-deterministic errors for debugging [13, 28].

Because of the versatilities, RnR frameworks are designed at various levels for different purposes, such as application level [13, 22, 30], OS level [3, 35], virtualization level [19, 27, 29]. Although RnR systems of Application and OS are more lightweight than VM RnR, they require modification of OS code or recompilation of the application, thus limited to open source-based operating system [11]. On the contrary, VM RnR systems are transparent to the guest OS system.

With the emerging of distributed architectures, more and more applications are deployed in VMC instead of the individual VM. For example, CyberGuarder [18] encapsulates security services into VMs and deploys them over a virtual network to provide virtual network security service. However, most previous studies focused on the individual VM [4], thus can not meet the requirements of analyzing distributed architecture. Some researchers devote themselves to achieving network traffic RnR and analyzing the behavior of distributed systems through network traffic [14, 20, 23, 31, 32], but it omits specific execution of terminal nodes, such as executions performed before sending or receiving a network packet. At the virtualization level, distributed snapshots of VMC $[6,7,15]$ record and replay the execution process of VMC by frequent global snapshots. However, it cannot reproduce the execution between two successive snapshots, 


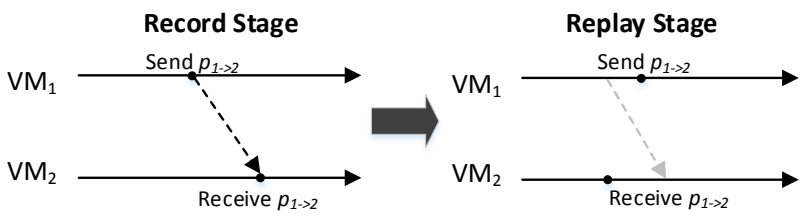

(a) The inconsistency caused by replay speed differential of VMs.

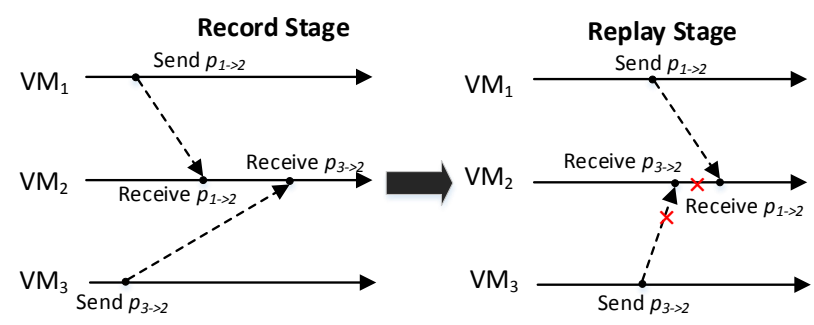

(b) The inconsistency caused by unreliable network environment.

Figure 1. The inconsistent states during VMC replay.

which is fatal in many cases, especially for security analysis, because some key execution processes might be omitted.

The RnR technology of VMC is an effective way to solve the above problems. First of all, it is OS-agnostic because it is implemented on the virtual machine manager (VMM). Meanwhile, it reproduces the system execution by instructions. However, there is no valid RnR framework for VMC, to our knowledge. If applying the existing RnR framework of individual VM $[19,26,28,29]$ to VMC, it would lead to an inconsistent global state or even replay crash. Therefore, we determine to achieve an $\mathrm{RnR}$ framework for VMC to replay the execution of VMC consistently.

The critical challenge for VMC RnR is consistency coordination. The leading causes of inconsistency are as follows. 1) Replay speed differential of VMs. The replay speed is not the same as normal execution and depends highly on its workloads. Therefore, the replay speed of VMs in the VMC is quite different from each other. As illustrated in Figure 1a, the $V M_{2}$ may runs faster than $V M_{1}$ during replaying. It results in that the receiving action of packet $p_{1->2}$ occurs before its sending action, which violates the principle of global consistency. 2) Unreliable network environment (e.g., network delay and packets loss). As illustrated in Figure 1b, due to the non-determinism of the network delay, the data packets arrive out of order in the record stage. However, the out-of-order behavior is non-reproducible in the replay stage, thereby changing the execution trajectory of the destination VM (i.e., $V M_{2}$ ) and making the replay fail.

The second problem is the overhead for events recording. For a VM, all received network packets are non-deterministic events that need to be logged, introducing heavy storage overhead. Figure 2 presents storage costs and events breakdown for different workloads deployed in VMC. For example,

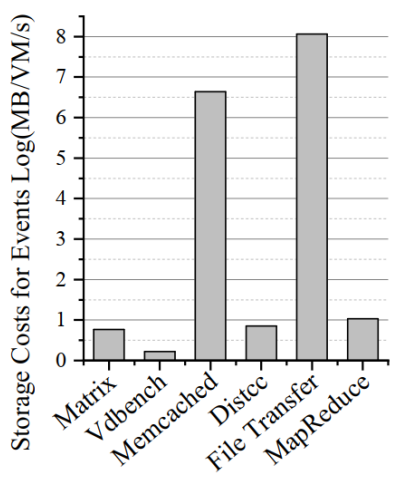

(a) Growth rate of log.

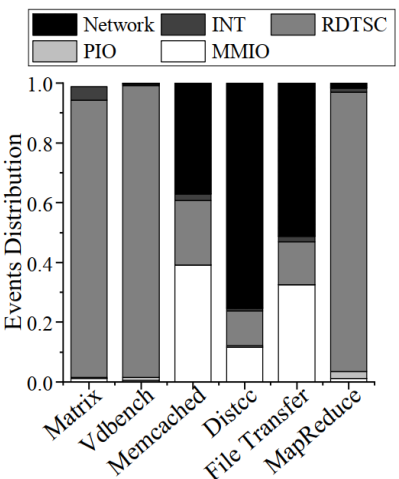

(b) Breakdown for events.
Figure 2. Overhead for non-deterministic events recording.

it consumes over $8 \mathrm{MB} / \mathrm{s}$ storage costs for File Transfer workload, of which network events account for $51.1 \%$. Therefore, frequent network interaction imposes heavy storage and performance overhead during recording, thus compromising the persistence and availability of the system.

To replay VMC deterministically and consistently, we propose ClusterRR, an RnR framework for VMC. It first integrates the replay of network events with the system execution of VMs, and proposes coordination protocols to schedule the replay process of all VMs through the timing of network events. Second, it employs a Hybrid RnR approach for network events RnR, i.e., applying the Order-based approach for reproducible internal network events and the Data-based approach for non-reproducible external network events, to mitigate overhead in the record stage. Moreover, we implement a network retransmission framework for network packets delivering during replaying to guarantee reproducibility.

ClusterRR is implemented in the VMM layer, so it is nointrusive and OS-agnostic for Guest OS and can work without other additional modules. In addition, it supports finegrained execution replay for VMC at the instruction level. To measure the effectiveness, we conduct experiments to compare it against VMC Snapshots (i.e., Hotsnap) in terms of performance and storage overhead. The results show that ClusterRR costs a much lower performance penalty and storage overhead than VMC Snapshot for compute-intensive and storage-intensive workloads. Moreover, compared to the native approach, the Hybrid approach mitigates the record overhead for network-intensive workloads. Specifically, there is a $66.9 \%$ reduction of storage costs and a $54.9 \%$ reduction of performance penalty for File Transfer.

In summary, our main contributions are as follows:

First, we propose an RnR framework for VMC and design coordination protocols to enable global consistency for deterministic replay $(\S 2.2)$.

Second, we employ a Hybrid RnR approach to mitigate the performance penalty and storage overhead (§2.3). 
Third, we implement a prototype ClusterRR and employ a network packets retransmission framework to ensure the correctness of packets replay $(\S 3)$.

Last, we conduct a set of experiments to demonstrate the effectiveness of our ClusterRR (§4).

\section{Design of ClusterRR}

In this section, we will first show the overall architecture of ClusterRR, then introduce how to coordinate global consistency in the record and replay stage. Next, we will describe the Hybrid RnR approach for network events.

\subsection{System Architecture}

ClusterRR is a RnR framework for whole VMC as illustrated in Figure 3. It consists of two parts, i.e., Record Component and Replay Component.

The Record Component is responsible for recording the execution of VMC. There exists a Recorder for every VM to log the execution of VM, including a Snapshoter to save instantaneous initial state and a Events Recorder to log nondeterministic events generated during subsequent execution. For VMC, the global consistency of all recorded VM states is another essential task. To coordinate consistency, it utilizes a global Record Coordinator to schedule all Recorders in the VMC, and employs a Controller in every Recorder to control the individual logging process according to global status.

In Replay Component, there is a Replayer in charging of individual VM replay, including reverting the VM state using Snapshot Loader and deterministically reproducing VM execution at instruction-level leveraging Events Replayer. In addition, just as Record Component, there also exists a global Replay Coordinator for replayed VMC and a Controller for every Replayer to coordinate global consistency. Moreover, to correctly replay network traffic, we implement Simulated NIC (sNIC) and Simulated Switch to deliver network packets generated by the replayed VM.

\subsection{Coordination Protocols of VMC Consistency}

In VMC, distributed programs are executed concurrently in different VMs, so there is no sequence for independent behaviors in different VMs. According to Lamport [16], the $\mathrm{VMC}$ is a partial ordering system. One approach to replay the VMC consistently is converting its execution into total ordering, but it would introduce heavy overhead. Another method is to mark the execution sequence by a global logic clock, but the global logic clock would limit performance due to contention. To mitigate the problem, we propose distributed coordination protocols for VMC record and VMC replay, i.e., Global Record Protocol and Global Replay Protocol, to achieve replay parallelly and consistently. In the following subsection, we will present some details.

2.2.1 Global Record Protocol. In the record stage, the logged state may be inconsistent caused by network delays.

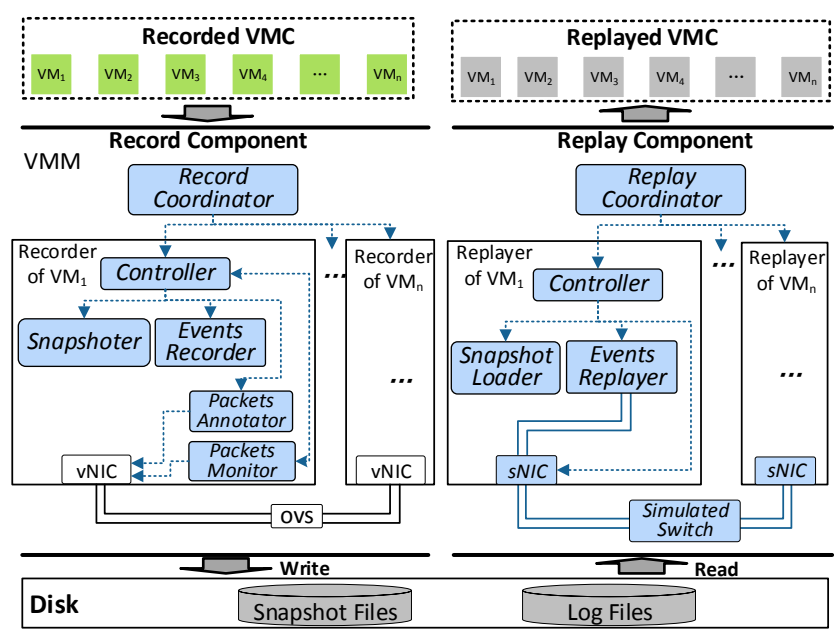

Figure 3. The architecture of ClusterRR.

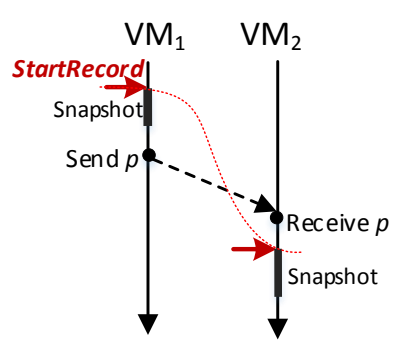

(a)

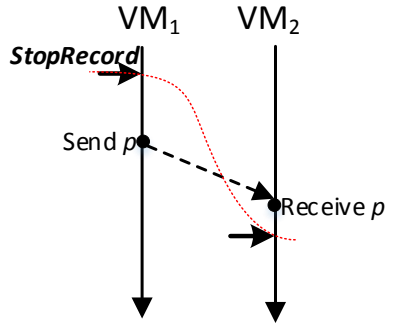

(b)
Figure 4. Recorded Inconsistent States. (a) Inconsistent state when conducting a VMC Snapshot. (b)Inconsistent state when stopping the record.

As illustrated in Figure 4a, the Record Coordinator broadcasts StartRecord command to every VMs to start recording. Once receiving the command, Snapshot is utilized to save the initial states of VMs. However, because of network delay, the arriving time of the StartRecord command to every VMs are different (i.e., as the red dotted line in the figure). If a network packet is transferring concurrently with the StartRecord broadcasting, the VM that has not started snapshot yet $\left(V M_{2}\right)$ may receive packets from VM that completed snapshot $\left(V M_{1}\right)$. Therefore, in the recorded states of VMC, the destination VM $\left(V M_{2}\right)$ has received the packet, but the source VM has not sent the packet. There also exists the same problem at the end of the recording, as shown in Figure 4b. Moreover, as shown in Figure 5b, some packet sending actions (e.g., $\left.p_{i(-1)}\right)$ will not be re-executed during replaying, but others (e.g, $\left.p_{i(2)}\right)$ will be re-executed and need to coordinate consistency. Therefore, for consistent replay, Recorder needs to distinguish the types of received network events and records them with different approaches. 


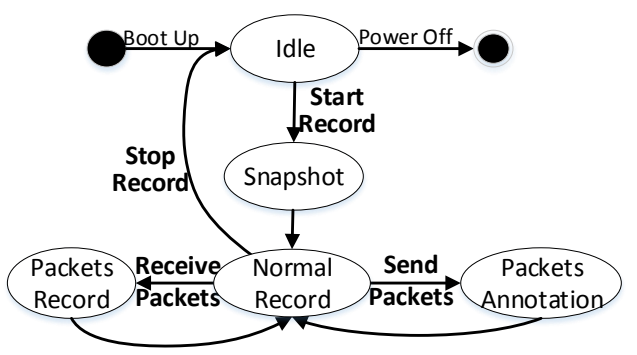

(a) Global Record Protocol.

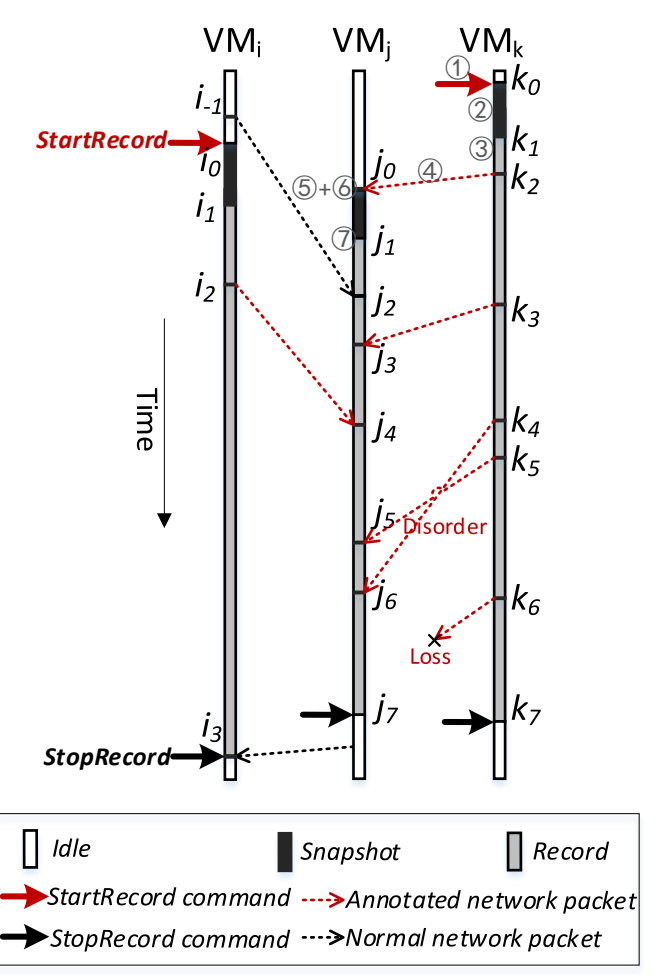

(b) An example. The symbol $m_{t}$ defines the time point $t$ of $V M_{m}$, and $p_{m(t)}$ is a network packet sent at this point.

Figure 5. The design of Global Record Protocol.

The main task of Global Record Protocol is to ensure the integrity and consistency of logged VMC state during recording. To achieve this goal, as illustrated in Figure 5a, it divides the record stage into the following five phases.

- Idle. The VMs in the VMC run normally, and their Controllers listen for messages from the global Coordinator.

- Snapshot. Snapshoter saves the instantaneous state of VM, including vCPUs, memory, disk and devices.

- Normal Record. Recorder captures non-deterministic events of VM execution and logs them into log files.

- Packets Annotation. To distinguish the types of network events, Packets Annotator in Recorder traps the network packets sending action and annotates outgoing network packets of recorded VM.
- Packets Record. When receiving packets, Packets Monitor in Recorder checks the type of the packets, and Events Recorder records the content and type of the packets into the log file.

Figure 5b presents an example of workflow of Global Record Protocol. To start a VMC record, Record Coordinator broadcasts the command of StartRecord (step 1). Once receiving this command, Controller of individual VM switches from Idle phase into Snapshot phase. The Controller immediately call Snapshoter to save instantaneous state of VM. It suspends the VM for a while and saves the state of vCPUs, memory, disk and devices of VM (step 2). After completing Snapshot, Controller switches the VM from Snapshot phase into Normal Record phase. In this phase, Events Recorder logs related non-deterministic events of the VM execution(step 3). Meanwhile, Packets Annotator annotates outgoing packets of recorded VM as red (step 4). Concurrently, Packets Monitor catches the incoming packets, gets their types and logs their information (step 5). If a VM in the phase of Idle receives a red network packet (e.g., $j_{0}$ ), it will enter an inconsistent state as illustrated in Figure 4a. To solve the inconsistency, Packets Monitor would buffer the packet and notify the Controller to start Snapshot immediately(step 6). After completing Snapshot, the Packets Monitor injects the packet and enters Normal Record phase(step 7).

Moreover, there may exist network delay, packet disordering $\left(p_{k(4)}, p_{k(5)}\right)$, and packet loss (e.g., $\left.p_{k(6)}\right)$. These nondeterministic factors are transparent to the Recorder because the behaviors of switches have not been recorded. To reproduce these behaviors during replaying, we employ Global Replay Protocol as will be described in the next subsection.

2.2.2 Global Replay Protocol. Global Replay Protocol is used for coordinating the global consistency of VMC states during the replay stage. The key idea of the protocol is to schedule the replay process of VMs according to the timing of network events. As illustrated in Figure 6a, it is divided into the following eight phases.

- Idle. There is the Controller for every VM that runs in idle, listening for messages from the Coordinators.

- Load Snapshot. Once receiving StartReplay command, the Snapshot Loader restores the VM state preparing for replay. Specifically, the Snapshot Loader suspends the VM and then loads the state of vCPUs, devices, disks and memory according to the snapshot files.

- Replay. The Events Replayer reads the content of nondeterministic events from the log file and injects them into replayed VM at the corresponding timestamp for deterministic replay.

- Packet Retransmission. When trapping a network packet sending action, the Replayer sends the identifier of the packet to the destination through simulated switches. 
- Judge. When receiving a network packet, the Replayer judges whether it is a packet from an unrecorded VM according to the recorded annotation.

- Mapping. Replayer scans the packets buffer to look for a packet with the same identifier as the subsequent network events.

- Inject. Replayer reads the packet from the log file, injects it into replayed VM, and continues the replay.

- Wait. Replayer suspends the VM to wait until receiving a packet from the recorded VM.

Figure 6b presents an example of Global Replay Protocol. To start a VMC replay, Replay Coordinator broadcasts the command of StartReplay to all Controllers (step 1). Once receiving this command, the Controller of individual VM switches from Idle into Load Snapshot phase and calls Snapshot Loader to restore the VM state preparing for replay (step 2). After completing Load Snapshot, the Controller enters the Replay phase. In the Replay phase, if there is no network event, the Replayer runs just like the replay of an individual VM, deterministically replaying the VM execution with no wait for others (step 3). Otherwise, consistency coordination is needed if there exist network interactions. When trapping a network packet sending action, the Replayer triggers Packet Retransmission. Specifically, it sends the identifier of the network packets to the destination through simulated switches. Then, simulated switches put them into packets buffer of the destination VM and notify the corresponding Replayer (step 4).

When replaying the receiving action of network events, the Replayer first judges whether the sending action of the events is out of the scope of recorded execution according to the recorded annotation(step 5). If so, Replayer directly enters Inject phase(step 6). If not, Replayer enters the Mapping phase, scanning the packets buffer to look for a packet with the same identifier as the subsequent network events (step 7). If Mapping fails, it enters the Wait phase to wait for the arrival of subsequent network packets, as $j_{3}$ (step 8). The Replayer suspends the VM to wait until receiving a packet from the recorded VM and enters the Mapping phase again (step 9), as $j_{3}^{\prime}$. If Mapping succeeds (e.g., $j_{3}^{\prime \prime}$ ), it indicates the corresponding packet sending action has been re-executed. Therefore, the Controller enters the Inject phase to replay packet receiving action (step 10).

The proposed Global Replay Protocol solves the inconsistency caused by the replay speed differential of VMs and the non-deterministic network events (i.e., network delay, packet disordering, and packet loss). As illustrated in Figure $6 \mathrm{~b}$, at the time point of $j_{3}$, Replayer knows the replay speed differential and network delay through Mapping and enters the Wait phase. Then, the replay would be suspended until receiving the exact network packet at the time point of $j_{3}^{\prime}$. For the replay of disordered packets, the packet that arrives in advance would be put into packets buffer at first, then

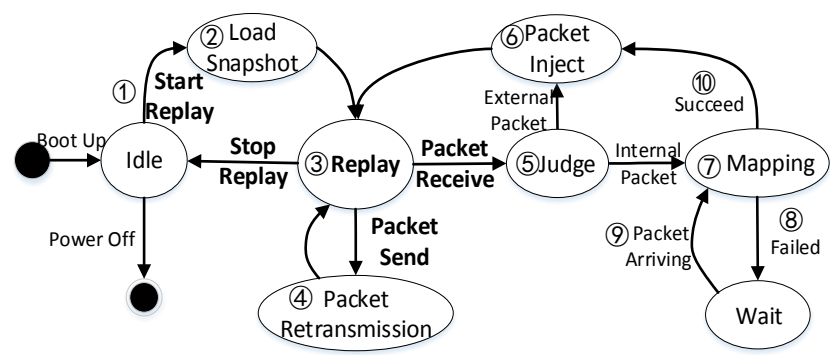

(a) Global Replay Protocol.
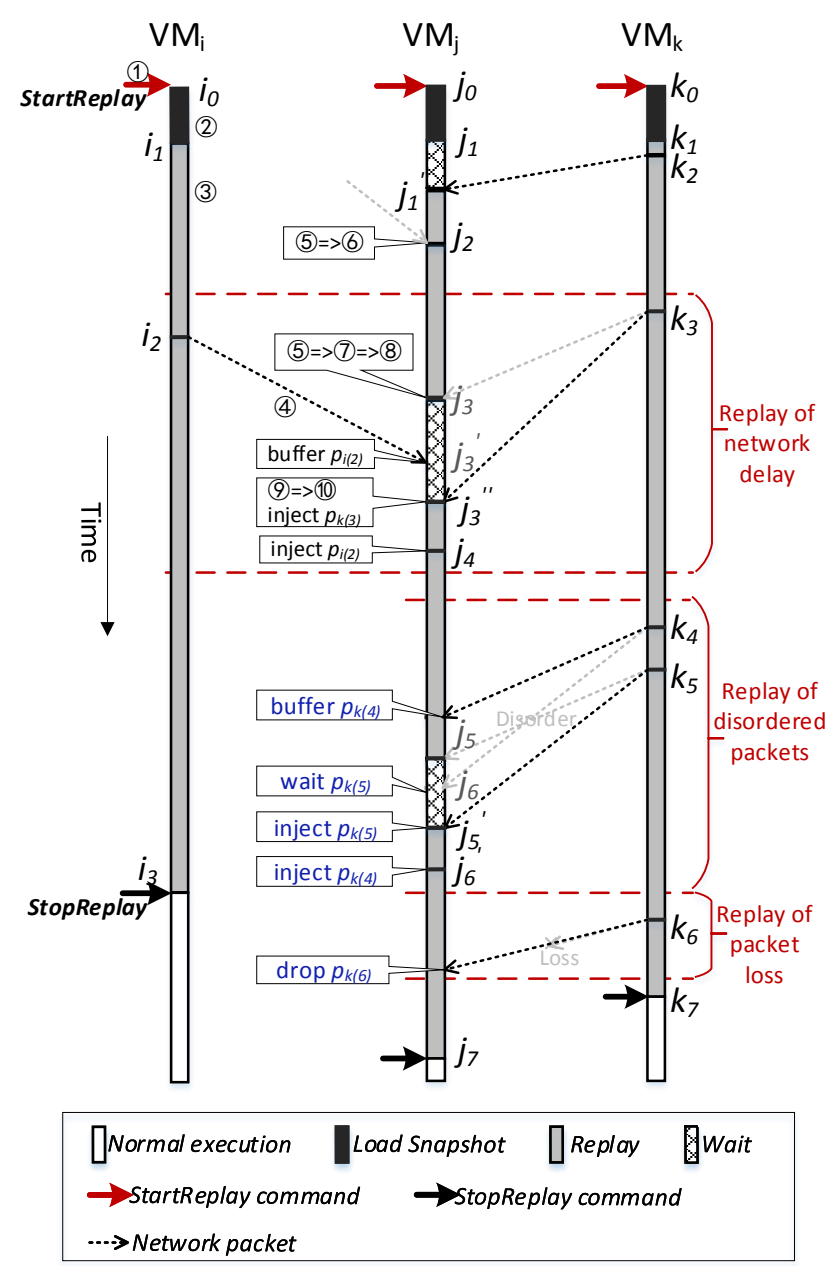

(b) An example. The symbol $m_{t}$ defines the time point $t$ of $V M_{m}$, and $m_{t}^{\prime}$ means the time point after suspending for a while. Meanwhile, $p_{m(t)}$ is the network packet sent by $V M_{m}$ at $m_{t}$.

Figure 6. The design of Global Replay Protocol.

be injected into replayed VM until reaching the recorded time point according to the Mapping and Inject mechanism. For example, as shown in Figure 6b, although packet $p_{k(4)}$ arrives at $V M_{j}$ earlier than packet $p_{k(5)}$, it would be buffered and injected into VM after the inject of $p_{k(5)}$ according to Global Replay Protocol, achieving consistent with recorded 
states. For the packet loss, since the behavior of network routing nodes is not replayed, the packets that should have been dropped by network routing nodes falsely arrives destination VM. According to the replay protocol, the Replayer would buffer these packets at first and drop them when exceeding the time threshold.

\subsection{Hybrid RnR Approach}

For individual VM, the content and arriving time of network packets are non-deterministic. Therefore, all their specific content and corresponding timestamps should be recorded into log files in the record stage. In the replay stage, Replayer reads the content from the log file to rebuild network packets and injects them into replayed VM. This approach is named as Data-based RnR approach. Unfortunately, for the RnR of VMC, it would introduce heavy performance and storage overhead when there are large amounts of information interactions and data transmissions among VMs.

We noticed that if we regard the recorded VMC as a whole, the recorded network events can be classified into two types, i.e., internal network events and external network events, as illustrated in Figure 7. Specifically, if a VM in the recorded VMC generates a network packet, the related events are called internal network events; otherwise, they are called external network events. For internal network events, their sending action would be reproduced deterministically during the replay stage. For example, at time $t$, the recorded $V M_{i}$ sends a network packet to recorded $V M_{j}$ with the specific content during the record stage. Then, in the replay stage, $V M_{i}$ would perform the same action as the record stage, sending network packets to $V M_{j}$ with the same packet content. It means that internal packets could be regenerated during the replay stage. Based on this observation, and also inspired by the subgroup replay approach of MPI applications[33], we propose a hybrid record and replay approach for network events to mitigate the overhead for network events recording, named as Hybrid RnR Approach.

The key idea of this approach is to classify network event streams into external network events and internal network events, then introduce the Data-based RnR approach and Order-based RnR approach, respectively. Specifically, as mentioned in $\$ 2.2 .1$, Recorder annotates the outgoing network packets sent by the recorded VM as red. Therefore, it could distinguish the internal network from external network packets in the record stage according to the annotation when receiving network packets. If it is an external network packet, Recorder records its specific content. Otherwise, the Recorder would only log the identifier of the packet (i.e., <source $M A C$, identification of IP header, flags, fragment offset>.

In the replay stage, different approaches are employed to replay external network events and internal network events. For external network events, their sending action would not be re-executed, so Replayer utilizes the Data-based approach to replay its receiving action, i.e., reading packets from the

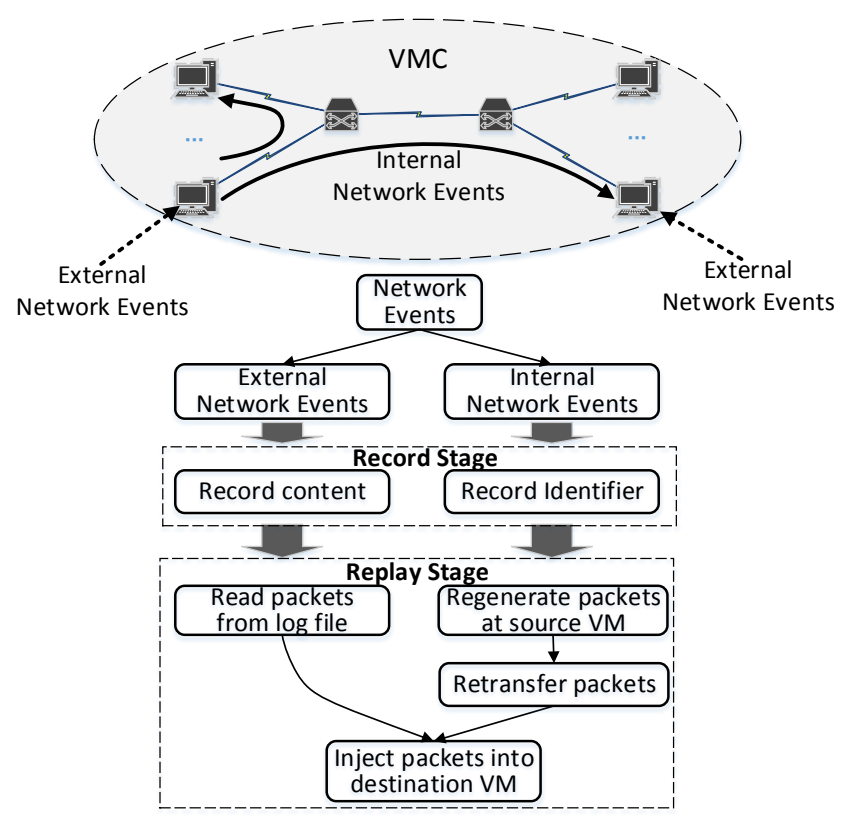

Figure 7. The schematic diagram of Hybrid RnR approach.

$\log$ file and injecting them into replayed VM. However, for internal network events, the source VM would regenerate the packets and re-execute the sending action. Because we have not replayed the behavior of network routing nodes, the network environment during replaying is likely to be different from the original execution, which would affect the reproducibility. Therefore, we designed simulated NIC and Simulated Switch to correctly delivery regenerated internal packets. The Replayer of source VM intercepts these packets and transmits them into destination VM through simulated NIC and Simulated Switches. Once receiving the expected packet, the Replayer would reinject it into the kernel of replayed VM. The specific details will be introduced in 3.2. In this way, the Hybrid RnR Approach reduces the penalty caused by recording the specific content of internal network events.

\section{Implemention}

We implement ClusterRR based on Qemu-1.5.3 and Linux3.10.0, adding $\sim 2900$ LOCs in Linux kernel for record and replay of individual VM, 1500 LOCs in Qemu for global consistency coordination, and $\sim 800$ LOCs in user space for network packets retransmission. In this section, we will present some implementation details.

\subsection{RnR Technology of Individual VM}

The VMC RnR is composed of the re-execution of all VMs and the global coordination of all replay processes. The coordination protocols have been discussed in $§ 2.2$. This subsection will present the RnR technology of individual VM. It enables to deterministically reproduce the execution of individual 
$\mathrm{VM}$ at the instruction level by logging non-deterministic events related to its execution in the record stage.

In the record stage, the Events Recorder records the nondeterministic events into the log file concurrently with the VM execution. Specifically, the non-deterministic events are classified into three types, Synchronous Events, Asynchronous Events, and Compound Events. For different types of events, they are recorded in various manners. 1) Synchronous Events, such as I/O events and RDTSC instructions, are issued by the guest VM to devices. These events would be triggered during the replay stage by associated instructions. In other words, these events occur deterministically as long as their previous instructions are executed as original execution. However, the contents of Synchronous Events are non-deterministic. Therefore, only the contents of these events need to be recorded. 2) Asynchronous Events, such as external interrupts, are generated from external devices. These events may occur at an arbitrary time, and their contents are non-deterministic, so both occurrence time and content should be logged. 3) Compound Events are events whose time and content are non-deterministic but are tied to Synchronous Events or Asynchronous Events. The network packet is an example. Its arrival time and payload are nondeterministic, but its arrival will first trigger an interrupt, so it is tied to an interrupt event. Thus it would be replayed following the injection of the associated interrupt. Therefore, the Recorder only needs to record the content of these events.

During the replay stage, to replay the execution of VM deterministically, Events Replayer reads the non-deterministic events from the log file and reinjects them into the VM at the appropriate time point to reproduce the execution by instruction. For different types of events, they are injected in different ways. For Synchronous Events, the Replayer only needs to fill the corresponding area with the logged content when they are triggered. For Asynchronous Events, the Replayer should wait until the VM execution reaches the logged timestamp and inject the events into the VM. For Compound Events, the Replayer injects them following the injection of events to which it is tied.

\subsection{Network Packets Retransmission}

To avoid interfering with machines out of the recorded VMC scope, the Record Component would not log the behavior of the routing nodes in the record stage. Therefore, the Replay Component is unable to replay their behavior in the replay stage. However, the network environment is constantly changing, so it is unlikely to be the same with the record stage, which would affect the reproducibility of VMC. Therefore, in the replay stage, how to retransmit the internal network packets as original execution is a problem.

In this subsection, we implement a framework for network packets retransmission to delivery network packets based on the execution of terminal nodes. It mainly consists a of Simulated NIC for each guest VM and a Simulated Switch for

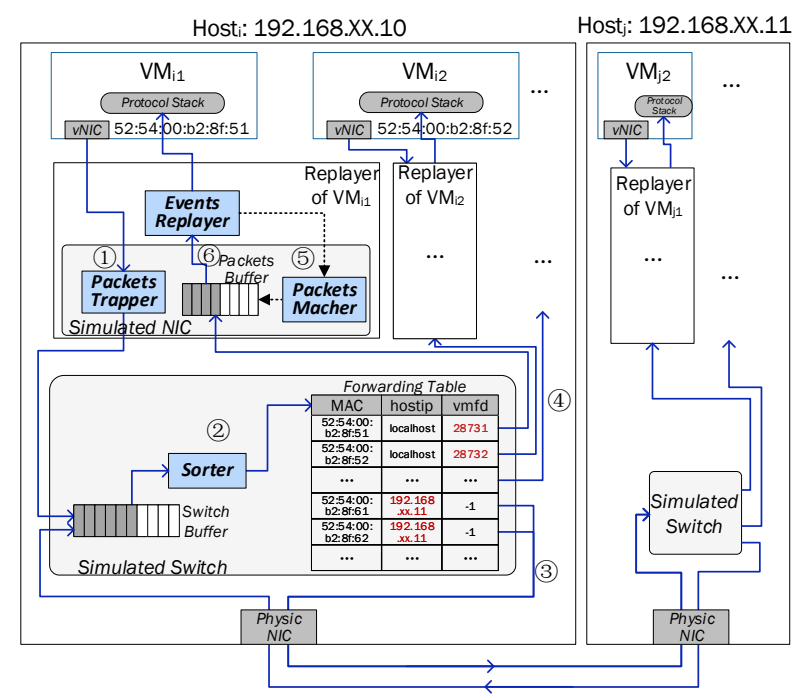

Figure 8. The framework of network packets retransmission.

each physic machine as shown in Figure 8. Simulated NIC plays the roles of original virtual NIC (vNIC), including Packets Trapper, Packets Buffer, and Packets Matcher. On the one hand, it traps network packets generated by VM and delivers them to Simulated Switch. On the other hand, it buffers network packets from other VMs for replay. Simulated Switch is composed of a Switch Buffer, Sorter and Forwarding Table. It is responsible for delivering received network packets from all VMs to the corresponding destination, including local VMs and remote Simulated Switches.

As shown in Figure 8, the retransmission workflow of network packets is divided into the following six steps. First, once network packets are regenerated by replay VMs, they would be trapped by Packets Trapper of Simulated NIC module and delivered to Switch Buffer (Step 1). Then, there is a Forwarding Table in each Simulated Switch to deliver packets to correct destinations. Different from traditional forwarding table, the Forwarding Table of Simulated Switch is a hash table storing hostip and $v m f d$ of all VMs in the recorded VMC and uniquely identifies each VM using its MAC address. Incessantly, Sorter of Simulated Switch parses the destination MAC of packets and looks for Forwarding Table to get corresponding hostip and $v m f d$ (step 2). If hostip is remote, the packets would be forwarded to remote Simulated Switch (step 3). Otherwise, they would be forwarded to corresponding Simulated NIC of destination VM in localhost through the $v m f d$ (step 4). Next, the received network packets are buffered in Packets Buffer of Simulated NIC, waiting to be chosen by Events Replayer. The Events Replayer sends the list of expected network packets identified by <source $M A C$, identification of IP header, flags, fragment offset $>$ to Packets Macher during replaying. Packets Macher finds corresponding packets and returns their instants to Events Replayer (step 
5). Finally, Events Replayer gets expected network packets through the returned instant and reinjects them into the kernel of replayed VM (step 6). Through this framework, the regenerated network packets would be correctly delivered from the source VM to the destination VM, so as to drive the execution of the Replayer.

\section{Evaluation}

In this section, we evaluate ClusterRR by a set of experiments. First, we measure the overhead of ClusterRR in the record stage and replay stage. Then, we demonstrate the gain of the Hybrid RnR approach compared to the native approach.

\subsection{Experimental Setup}

All experiments are conducted on physical servers configured with a 2 way 6 core Intel $2.30 \mathrm{GHz}$ CPU, $64 \mathrm{~GB}$ of RAM, and 1 TB SSD running Centos 7 with 3.10.0-693.el7.x86_64 kernel. We configure the VM with one vCPU and 2 GB RAM running Ubuntu 16.04. In addition, the VMC for the experiment consists of eight VMs unless specified otherwise.

Our ClusterRR is the first RnR framework for VMC, and it offers execution replay at the instruction level, which solves the problem of missing execution details when using VMC Snapshot to replay VMC behavior for security analysis. Therefore, we compare ClusterRR with Hotsnap[6], a VMC Snapshot approach, in terms of performance and storage costs. Moreover, we compare our ClusterRR with the RnR framework of individual VM without the constraints of coordination protocol (i.e., Baseline).

Moreover, to evaluate ClusterRR thoroughly, we deploy the following workloads in the VM to represent a variety of characteristics. (1) Matrix multiplies two randomly generated square matrices. This workload is both memory-intensive and CPU-intensive. (2) Vdbench is a disk I/O workload to be used for measuring storage performance and storage data integrity. (3) File Transfer is a distributed application we designed to transfer files between two VMs in pairs concurrently. In this section, we transfer a $600 \mathrm{M}$ file. (4) Distcc is a distributed compiler, which distributes $\mathrm{C} / \mathrm{C}++$ compilation tasks to the VMs in VMC, and the results are the same as a local compilation. (5) MapReduce is a parallel computing model for large-scale data processing proposed by Google. (6) Memcached is an in-memory key-value store for small chunks of data. We set the Memcached server in two VMs and configure Twemperf[1] as the clients in other VMs to randomly request data.

\subsection{Correctness of ClusterRR}

Correctness means ClusterRR correctly records all execution of the running VMC, and recorded execution can be reproduced correctly and deterministically as original execution. To verify the correctness, we conduct experiments as follows. First, we compile Qemu leveraging the distributed compiler

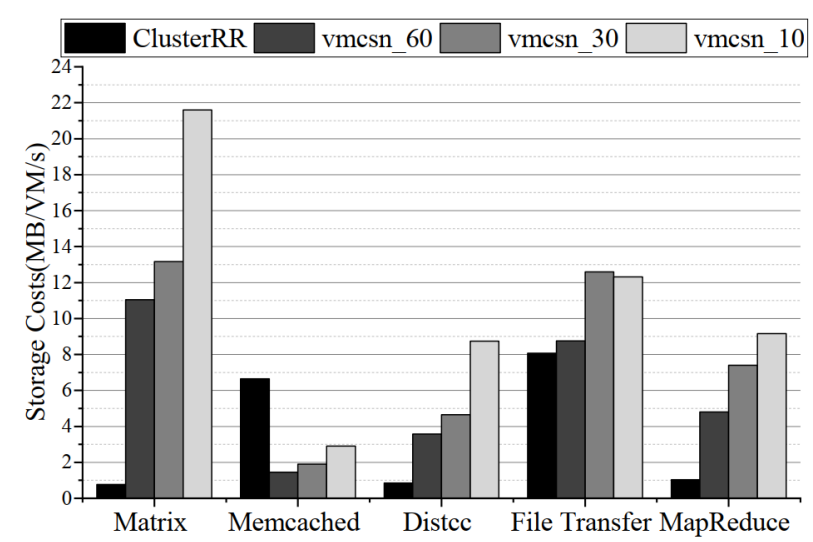

Figure 9. Storage costs of ClusterRR and VMC Snapshot.

Distcc and $\log$ the execution of VMC. Then, we replay the logged execution for a while and then stop the replay. After the replay is stopped, the Distcc application continues its execution, and the compiled Qemu can execute successfully. Moreover, we transfer files between VMs during recording. When the replay is complete, the destination VMs get the same file as the original execution. These results demonstrate the correctness of our system.

\subsection{Overhead of VMC Record}

In this subsection, we conduct experiments to measure the overhead of ClusterRR in the record stage. Firstly, we deploy various workloads in the VMC and record its execution leveraging VMC Snapshot (i.e., Hotsnap) and ClusterRR respectively. When using VMC Snapshot, we configure it with varying snapshot intervals, i.e., $60 \mathrm{~s}$ (vmcsn_60), $30 \mathrm{~s}$ (vmcsn_30), or $10 s$ (vmcsn_10). Then we compare them in terms of storage costs and performance loss.

4.3.1 Storage Overhead. Figure 9 depicts the growth rate of storage costs when using different record mode. The results show that most of the storage costs of ClusterRR are much less than those of VMC Snapshot under the experimental settings. For example, Matrix introduces the maximum storage costs for VMC Snapshot, i.e., over $11 \mathrm{MB} / \mathrm{VM} / \mathrm{s}$ in all cases, while it incurs the least storage costs when using ClusterRR i.e., $0.766 \mathrm{MB} / \mathrm{VM} / \mathrm{s}$. It is because Matrix incurs fewer non-deterministic events for ClusterRR to be recorded. In contrast, Matrix consumes a significant amount of memory to calculate and the memory changes instantaneously, so VMC Snapshot costs a lot to save the variation of VM memory. Moreover, the storage costs of VMC Snapshots tend to increase with the interval decreasing. For example, the storage costs increase from $11.0 \mathrm{MB} / \mathrm{s}$ to $21.6 \mathrm{MB} / \mathrm{s}$ when the interval decreases from the $60 s$ to $10 s$ for Matrix. Different from others, the storage costs of ClusterRR are higher than VMC Snapshots for Memcached workload, and those of the 


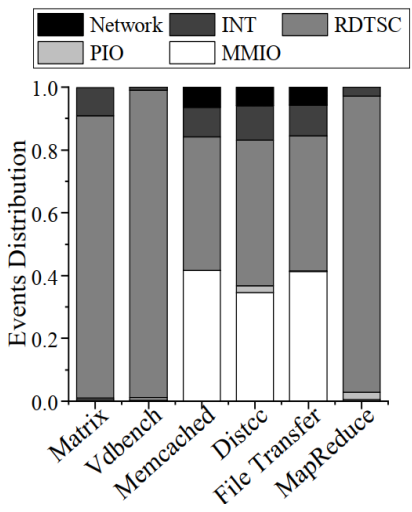

(a)

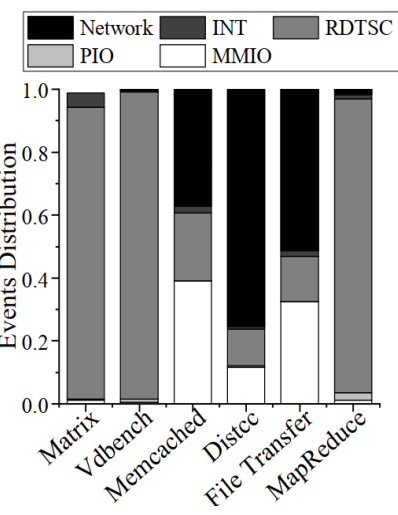

(b)
Figure 10. Breakdown for non-deterministic events. (a) By count. (b) By storage overhead.

two are similar for File Transfer. To analyze the factor, we calculate the breakdowns for non-deterministic events by count and storage costs, then report them in Figure 10. According to the results, Memcached and File Transfer workloads involve a large number of I/Oes. Especially for network events, although it only accounts for a small proportion as shown in Figure 10a, it costs a large percentage of storage as shown in Figure 10b. For example, for Memcached, network events only account for $5.57 \%$ but cost $51.2 \%$ storage overhead. It is because the full content of network packets should be logged. To mitigate this problem, we propose Hybrid RnR Approach, and we will measure it in $§ 4.5$.

4.3.2 Performance Penalty. To measure the performance penalty, we normalize the execution time of workloads by the execution time during normal execution and report the results in Figure 11. We see that the performance of ClusterRR is better than that of VMC Snapshots in most cases, especially when the interval of snapshots is $10 \mathrm{~s}$. It is worth noting that ClusterRR could record execution at the instruction level. However, for VMC Snapshot, the snapshot interval should be further reduced if the fine-grained recording is required, introducing a more significant performance penalty.

\subsection{Performance of VMC Replay}

In this subsection, we measure the replay performance of ClusterRR. We first evaluate the replay performance when the network scale varies from $2 \mathrm{VMs}$ to $32 \mathrm{VMs}$ (The experiments configured with more than $16 \mathrm{VM}$ were performed on two hosts). Then, we analyze the causes of the replay penalty by comparing it with individual VM (i.e., Baseline).

Figure 12 depicts the replay performance of ClusterRR with varying VMC scales. With increasing VMC scale, the replay time decreases for Distcc and File Transfer, while it increases for Memcached. To analyze the cause, we replay the

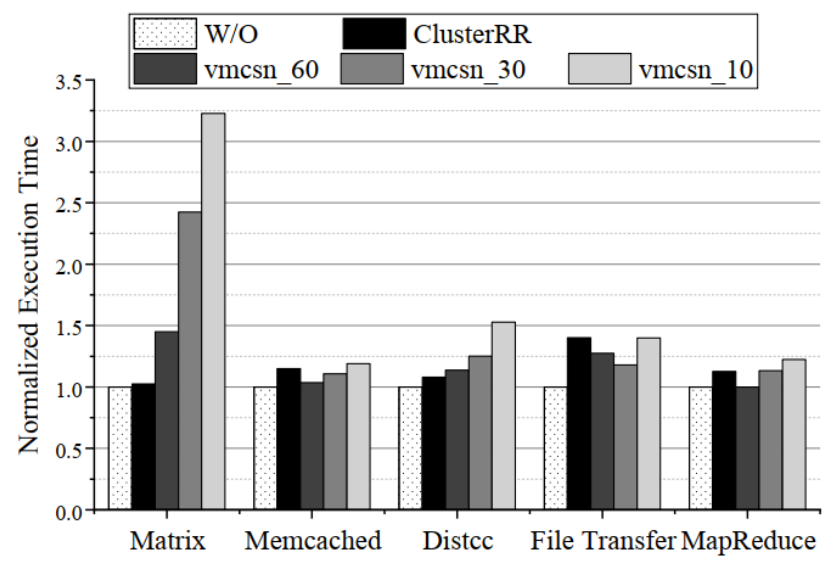

Figure 11. Performance penalty of ClusterRR vs. VMC Snapshot in the record stage.

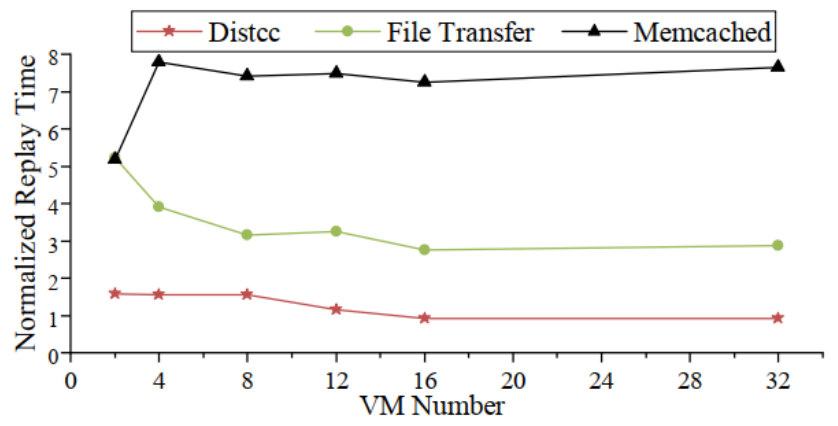

Figure 12. Replay performance.

same VMC execution process without consistency coordination (i.e., Baseline), logs the replay time of VMs separately, and report the results in Fig 13.

On the whole, the replay time of Baseline is shorter than that of ClusterRR, and the extra time for ClusterRR is mainly caused by consistency coordination. In addition, under our experimental setting, the smaller the network scale, the greater the cost of coordination. Moreover, the replay time of Baseline and ClusterRR tends to be close and stable as the network scale increases. The reason is as follows. For Memcached, with increasing network scale, the performance of servers would be limited by the hardware resources. Meanwhile, the response of the server becomes slower, and the workload of clients decreases, which causes the replay of clients to become much faster than servers, according to the results of Baseline. Therefore, though constrained by coordination protocols, there is little time for servers to wait for the execution of other VMs, so the replay time of the Baseline and ClusterRR tends to be the same. For Distcc, initially, as the number of nodes increases, the compilation tasks allocated to each node will decrease. Therefore, the replay time tends to be shorter. When the VMC scale exceeds a threshold, some of the VM would not be used under 


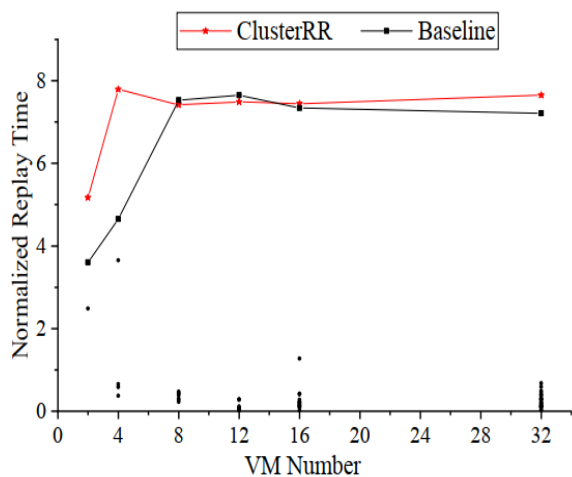

(a) Memcached

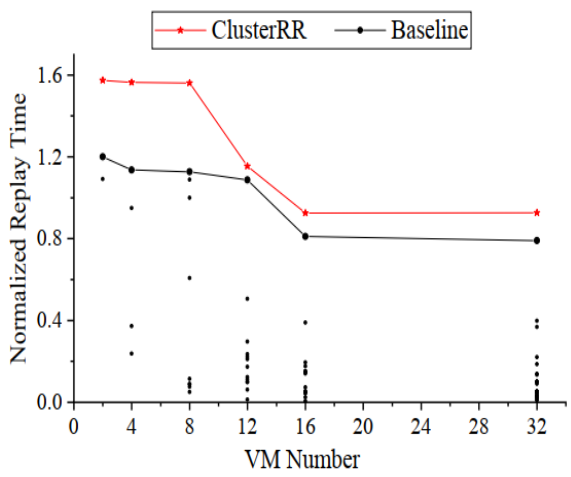

(b) Distcc

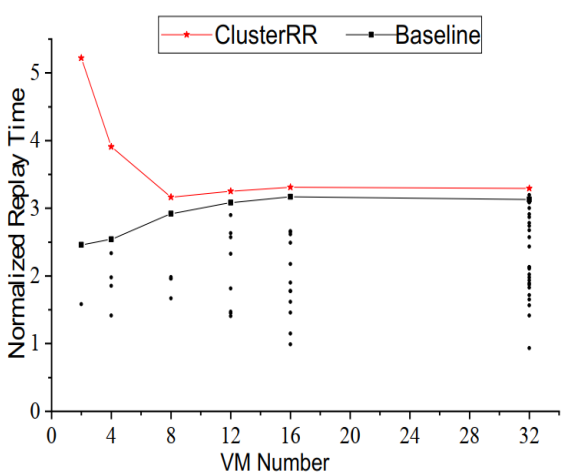

(c) File Transfer

Figure 13. Replay performance of ClusterRR vs. Baseline. ClusterRR coordinates global consistency for the VMC, so all VMs finish replaying almost at the same time. We present the overall time in this figure. Baseline replays VMs independently and pays no attention to global consistency, so replay time is different from each other. In the figure, we connect the longest replay time of all modes to a line graph, and the rest of the results are presented in the form of scattered points.

the set compilation command. Therefore, the replay time becomes steady. For File Transfer, when the network scale is smaller than $4 \mathrm{VM}$, the replay time of ClusterRR is much longer than Baseline (about $2 \times$ ), caused by the consistent replay protocol. With the increasing of VMs, the replay time of ClusterRR decreases as bandwidth speed falls caused by hardware resources limitation. For the recorded VMs, the number of executed instructions in each time unit is reduced relatively, so the time required for the replay is reduced. In conclusion, many factors affect replay performance, including consistency coordination and load conditions on VMC. According to the experiment results, the impact of consistency coordination on replay performance is much smaller than the impact of VMC workload on it.

\subsection{Gains of Hybrid RnR Approach}

To measure our Hybrid RnR approach, we deploy various workloads in the VMC, record its execution leveraging the native Data Record approach and our Hybrid Record approach, and replay with the corresponding replay method. Then, we compare these two approaches in terms of storage overhead, performance penalty, and replay performance.

4.5.1 Storage Overhead in the Record Stage. Figure 14 depicts the storage costs of VMC Record with different record approaches. As expected, the storage costs of the Hybrid Record are less than those of Data Record, especially for network-intensive workloads. For example, the storage costs decreases by $27.4 \%$ (i.e., $(6.64-4.82) / 6.64)$ for Memcached, $43.5 \%$ (i.e., $(0.85-0.48) / 0.85)$ for Distcc, and $66.9 \%$ (i.e., $(8.06-2.67) / 8.06)$ for File Transfer. According to the storage breakdown for specific events, the decrease in storage consumption is mainly due to network events. The reason is that Hybrid Record only logs the identifier of the internal

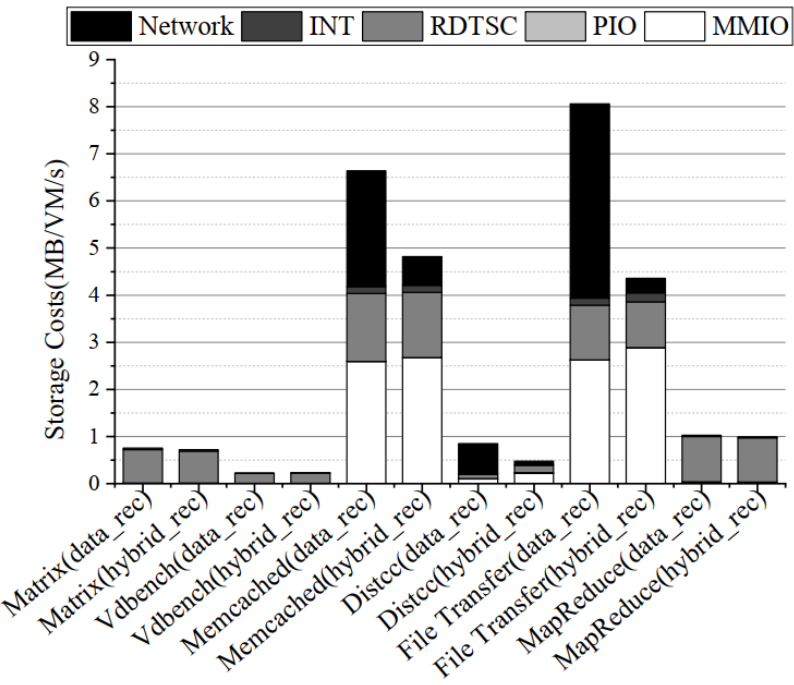

Figure 14. Storage overhead of Data Record vs. Hybrid Record.

network packets instead of the entire contents. Therefore, the experiment results prove that our Hybrid Record could significantly reduce the storage costs caused by network communication within the VMC. Moreover, we have noticed that the storage cost of MMIO events slightly increases when using the Hybrid Record approach. It is probably because the Hybrid approach mitigates the performance penalty, which will be analyzed in the following subsection.

4.5.2 Performance Penalty in the Record Stage. We calculate the performance penalty by the ratio of extra time costs to the execution time without VMC Record and report the results in Tab.1. As expected, Hybrid Record mitigates the performance penalty caused by network events logging. 
Table 1. Performance penalty of Hybrid Record vs. Data Record.

\begin{tabular}{ccl}
\hline WorkLoads & Data Record & Hybrid Record \\
\hline Matrix & $2.60 \%$ & $2.56 \%$ \\
vdbench & $24.41 \%$ & $2.38 \%$ \\
Memcached & $15.08 \%$ & $9.76 \%$ \\
Distcc & $8.09 \%$ & $6.47 \%$ \\
File Transfer & $51.76 \%$ & $23.30 \%$ \\
MapReduce & $18.75 \%$ & $7.14 \%$ \\
\hline
\end{tabular}

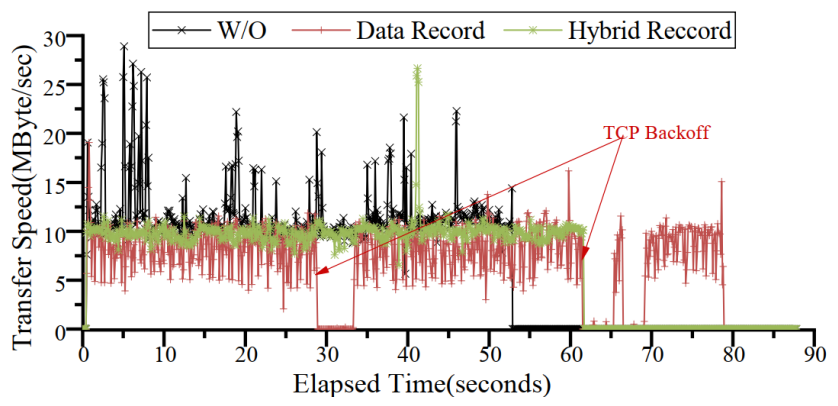

Figure 15. The network transfer speed under modes of Data Record vs. Hybrid Record.

For example, the performance penalty decreases from $15.08 \%$ to $9.76 \%$ for Memcached, and $8.09 \%$ to $6.47 \%$ for Distcc, respectively. Especially for File Transfer workload, it reduces by $54.9 \%$ (i.e., $(51.7 \%-23.3 \%) / 51.7 \%$ ).

To analyze the reasons for performance improvement, we run a VMC consisting of $16 \mathrm{VMs}$ and transfer files $(600 \mathrm{M})$ in pairs under these two Record modes. During transferring, we $\log$ transfer speed every $0.1 s$ and report the results in Figure 15. It can be seen that there are TCP backoffs in Data Record mode caused by packet loss. It is because the File Transfer not only generates a large number of $\mathrm{I} / \mathrm{O}$ operations on the VM but also triggers a large number of interrupt, I/O, and network events to be logged as shown in Figure 14. Therefore, the Recorder process would compete for I/O resources with normal applications. In this situation, the Recorder is prone to be blocked because of running out of write buffer, leading to VM suspension and network packet loss.

Furthermore, we measure the performance penalties of these two record approaches under different scales of VMC and report the results in Figure 16. The results show that the performance penalties of both approaches increase because $\mathrm{I} / \mathrm{O}$ competition becomes more and more fierce as the network scale increases, but the performance penalty of Data Record increases more obviously than that of Hybrid Record. For example, the reduction of performance penalty is $3.1 \%$ (i.e., $17.3 \%-14.2 \%$ ) for $2 \mathrm{VMs}$ case, and it increases to $28.0 \%$ (i.e., $51.7 \%-23.3 \%$ ) for $8 \mathrm{VMs}$ case. We also notice that the performance penalty decreases when the scale of VMC is

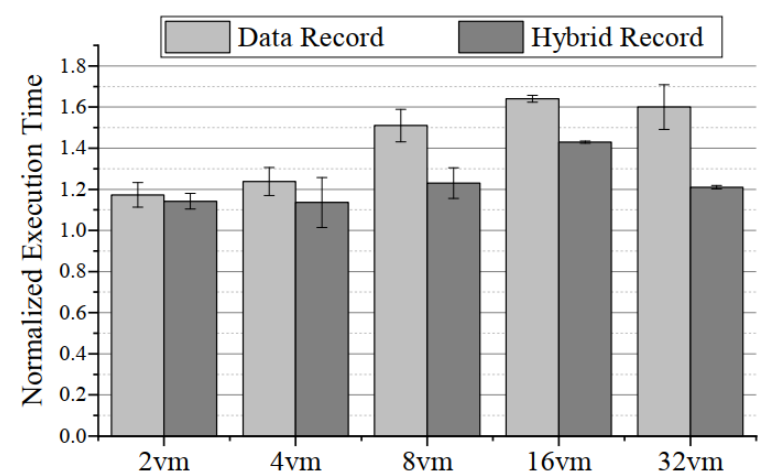

Figure 16. The performance penalty of Data Record vs. Hybrid Record with vaious number of VMs.

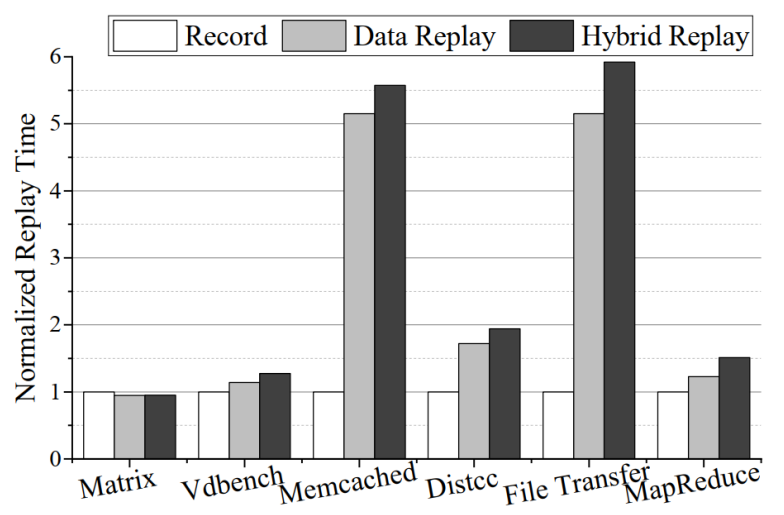

Figure 17. Replay performance with various workloads.

over $16 \mathrm{VMs}$. It is probably because the performance degradation caused by resource competition is more prominent than the performance penalty caused by VMC Record. In general, Hybrid Record approaches could mitigate the performance penalty for network-intensive workloads.

4.5.3 Replay Performance. Figure 17 shows the replay time normalized by the normal execution time. It can be seen that the Hybrid Replay takes a little longer than Data Replay. There are two main reasons. On the one hand, the Hybrid Replay transmits the entire content of the network packet as normal execution, while the Data Replay only transmits the identifiers of packets. Therefore, the Hybrid Replay leads to longer network delay. On the other hand, according to the results above, the performance penalty of Hybrid Record is lower than that of Data Record in the record stage, so more instructions are executed in the same time slice. Therefore, more time is required to replay. Although File Transfer causes the most significant extra replay time $0.77 \times$ (i.e., $(5.92-5.15) \times)$ under our experimental setting, it is still acceptable compared to its replay time (i.e., 5.15X). Moreover, the timeliness requirement of the replay stage is much lower than that of the record stage. 


\section{Related Work}

Record and Replay (RnR) systems have been researched and applied in many fields. They can be classified into following categories: application level [11, 12, 24], OS kernel level [3, 35], network traffic level [20, 21, 23] and VM level [25-29].

At the application and OS kernel level, researchers are mainly devoted to optimizing the record performance of multicore processors and coordinating replay of distributed applications. For example, PRES [24] reduces the record overhead by omitting partial non-deterministic information at the cost of multiple coordinated replays. RelaxReplay [12] proposes a new way of capturing memory access reordering and building an RnR system for any relaxed-consistency model. Moreover, there are some researchers on distributed applications. For the replay of distributed applications, liblog [11] embeds 8 byte Lamport clocks to timestamp all outgoing messages during execution and then use these virtual clocks to schedule replay. To reduce the trace size, Babaei et al. [2] proposes an approach to collecting and reordering execution traces that do not rely on timestamps.

Another group of researchers focuses on the replay of network traffic. For example, OFRewind [32] proposes the first replay tool for debugging network domains. Moreover, Mahimahi [23] achieves a framework to record traffic from HTTP-based applications and replay it under emulated network conditions. ProxyReplay [14] replays application-layer traffic for network proxies. Huang et al. [31] proposes a traffic replay method based on interactive sequence and timestamp of packets to generate the network traffic. DETER [20] supports replaying selected TCP transactions to reproduce issues for diagnosis through capturing all the interactive traffic in a TCP connection all the time at all the hosts.

RnR systems of application, OS, and network traffic are more lightweight than VM RnR systems, but they usually need to modify or recompile the application or kernel. Thus, an open source-based operating system is required. Moreover, their behavior is perceived by the operating system. Therefore, they cannot resist attacks against the kernel for security analysis because they can be bypassed. Fortunately, RnR systems at the VM level replay the execution of the whole guest VM with no-intrusive and OS-agnostic because it runs in the VMM. ReVirt [9] replays the entire VM by recording all non-deterministic events based on the UMLinux platform. Aftersight [17] traces the execution flow of a VM based on the VMware hypervisor and then rebuilds it on QEMU emulation through a transformation. SMP-Revirt [10] implements the first deterministic replay system for a multiprocessor VM on commodity hardware leveraging CREW protocol. Lore [19] designs a logging and replay system based on KVM (Kernel-based Virtual Machine). Samsara [25-27] leverages the HAV extension to achieve efficient and practical deterministic replay for multiprocessor VM. RnR-Safe [29] accelerates the replay and diagnosis procedure by narrowing the range of detailed analysis.

However, previous VM-level work mainly focuses on the RnR of individual VM, including extending the platform, optimizing the performance, and applying the framework into security analysis. There is little work that has been done on distributed VMC. In the last two decades, VMC Snapshot has been studied for security analysis and failure recovery. It mainly focuses on designing the snapshot protocol between the peers to maintain a globally consistent state and reduce the performance penalty. For example, VNSnap [15] leverages Xen live migration function to minimize system downtime when taking VMN snapshots. HotSnap [6] designs a VMC Snapshot approach to enable taking hot distributed snapshots with milliseconds system downtime and TCP backoff duration. Although VMC Snapshots have the advantages of fast rollback, coarse-grained snapshots cannot support fine-grained behavior analysis, and frequent snapshots would introduce high performance and storage overhead.

Compared to previous works, our ClusterRR devotes to replay the execution of whole virtual machine clusters instead of individual VM. It designs coordination protocols and network packets retransmission framework to ensure global consistency and reproducibility of replay. Moreover, it proposes a Hybrid RnR approach to reduce record overhead by omitting specific content of internal network packets.

\section{Conclusion and Future Work}

This paper presents an RnR framework for a virtual machine cluster named as ClusterRR. To deterministically replay VMC, ClusterRR proposes coordination protocol, i.e., Global Record Protocol and Global Replay Protocol, to schedule the record processes and replay processes in VMC. In addition, it proposes a Hybrid RnR Approach for network events, thereby reducing the performance and storage overhead of non-deterministic events recording. Moreover, we implement a framework to retransmit network packets during replaying to guarantee reproducibility. Furthermore, a set of experiments demonstrate that our ClusterRR outperforms existing VMC Snapshot frameworks, and it could be useful in practical clouds and security analysis.

The goal of ClusterRR is to provide an efficient RnR framework for virtual machine clusters. At present, it only supports VM with one vCPUs and one network card. In the future, we will devote ourselves to extending ClusterRR to support VMs with multiple vCPUs and network cards.

\section{Acknowledgments}

This work was supported in part by the National Natural Science Foundation of China (Grant No. 62072453, 61972392) and the Youth Innovation Promotion Association of the Chinese Academy of Sciences (Grant No. 2020164). 


\section{References}

[1] 2014. Twemperf. https://github.com/twitter-archive/twemperf.

[2] Majid Babaei, Mojtaba Bagherzadeh, and Juergen Dingel. 2020. Efficient Reordering and Replay of Execution Traces of Distributed Reactive Systems in the Context of Model-Driven Development (MODELS '20). Association for Computing Machinery, 285-296. https: //doi.org/10.1145/3365438.3410939

[3] Arkaprava Basu, Jayaram Bobba, and Mark D. Hill. 2011. Karma: Scalable Deterministic Record-Replay. In Proceedings of the International Conference on Supercomputing (ICS '11). Association for Computing Machinery, 359-368. https://doi.org/10.1145/1995896.1995950

[4] Dylan Chapp, Kento Sato, Dong H Ahn, and Michela Taufer. 2018. Record-and-replay techniques for HPC systems: A survey. Supercomputing Frontiers and Innovations 5, 1 (2018), 11-30.

[5] Jim Chow, Dominic Lucchetti, Tal Garfinkel, Geoffrey Lefebvre, Ryan Gardner, Joshua Mason, Sam Small, and Peter M. Chen. 2010. MultiStage Replay with Crosscut. In Proceedings of the 6th ACM SIGPLAN/SIGOPS International Conference on Virtual Execution Environments (VEE '10). Association for Computing Machinery, New York, NY, USA, 13-24. https://doi.org/10.1145/1735997.1736002

[6] Lei Cui, Bo Li, Yangyang Zhang, and Jianxin Li. 2013. HotSnap: A Hot Distributed Snapshot System For Virtual Machine Cluster. In Proceedings of the 27th USENIX Conference on Large Installation System Administration (USENIX LISA '13). USENIX Association, 59-74. https://www. usenix.org/conference/lisa13/technical-sessions/presentation/cui

[7] Lei Cui, Jianxin Li, Tianyu Wo, Bo Li, Renyu Yang, Yinglie Cao, and Jinpeng Huai. 2014. HotRestore: A Fast Restore System for Virtual Machine Cluster. In 28th Large Installation System Administration Conference (LISA '14). USENIX Association, 10-25. https://www.usenix. org/conference/lisa 14/conference-program/presentation/cui

[8] Daniela A. S. de Oliveira, Jedidiah R. Crandall, Gary Wassermann, S. Felix Wu, Zhendong Su, and Frederic T. Chong. 2006. ExecRecorder: VMBased Full-System Replay for Attack Analysis and System Recovery. In Proceedings of the 1st Workshop on Architectural and System Support for Improving Software Dependability (ASID '06). Association for Computing Machinery, 66-71. https://doi.org/10.1145/1181309.1181320

[9] George W. Dunlap, Samuel T. King, Sukru Cinar, Murtaza A. Basrai, and Peter M. Chen. 2003. ReVirt: Enabling Intrusion Analysis through Virtual-Machine Logging and Replay. SIGOPS Oper. Syst. Rev. 36, SI (2003), 211-224. https://doi.org/10.1145/844128.844148

[10] George W. Dunlap, Dominic G. Lucchetti, Michael A. Fetterman, and Peter M. Chen. 2008. Execution Replay of Multiprocessor Virtual Machines. In Proceedings of the 6th ACM SIGPLAN/SIGOPS International Conference on Virtual Execution Environments (VEE '08). Association for Computing Machinery, 121-130. https://doi.org/10.1145/1346256. 1346273

[11] Dennis Geels, Gautam Altekar, Scott Shenker, and Ion Stoica. 2006. Replay Debugging for Distributed Applications. In Proceedings of the Annual Conference on USENIX'06 Annual Technical Conference (ATC '06). USENIX Association, USA, 27.

[12] Nima Honarmand and Josep Torrellas. 2014. RelaxReplay: Record and Replay for Relaxed-Consistency Multiprocessors. In Proceedings of the 19th International Conference on Architectural Support for Programming Languages and Operating Systems (ASPLOS '14). Association for Computing Machinery, 223-238. https://doi.org/10.1145/2541940.2541979

[13] Nima Honarmand and Josep Torrellas. 2014. Replay debugging: Leveraging record and replay for program debugging. In 2014 ACM/IEEE 41st International Symposium on Computer Architecture (ISCA '14). 455-456. https://doi.org/10.1109/ISCA.2014.6853229

[14] Chun-Ying Huang, Ying-Dar Lin, Peng-Yu Liao, and Yuan-Cheng Lai. 2015. Stateful traffic replay for web application proxies. Security and Communication Networks 8, 6 (2015), 970-981. https://doi.org/10.1002/ sec. 1053
[15] Ardalan Kangarlou, Patrick Eugster, and Dongyan Xu. 2009. VNsnap: Taking snapshots of virtual networked environments with minimal downtime. In 2009 IEEE/IFIP International Conference on Dependable Systems Networks (DSN '09). 524-533. https://doi.org/10.1109/DSN. 2009.5270298

[16] Leslie Lamport. 2019. Time, clocks, and the ordering of events in a distributed system. In Concurrency: the Works of Leslie Lamport. 179-196.

[17] Dongyoon Lee, Benjamin Wester, Kaushik Veeraraghavan, Satish Narayanasamy, Peter M. Chen, and Jason Flinn. 2010. Respec: Efficient Online Multiprocessor Replayvia Speculation and External Determinism. In Proceedings of the Fifteenth International Conference on Architectural Support for Programming Languages and Operating Systems (ASPLOS '10). Association for Computing Machinery, 77-90. https://doi.org/10.1145/1736020.1736031

[18] Jianxin Li, Bo Li, Tianyu Wo, Chunming Hu, Jinpeng Huai, Lu Liu, and KP Lam. 2012. CyberGuarder: A virtualization security assurance architecture for green cloud computing. Future generation computer systems 28, 2 (2012), 379-390. https://doi.org/10.1016/j.future.2011.04. 012

[19] Jianxin Li, Shouyu Si, Bo Li, Lei Cui, and Jingsheng Zheng. 2013. LoRe: Supporting Non-deterministic Events Logging and Replay for KVM Virtual Machines. In 2013 IEEE 10th International Conference on High Performance Computing and Communications \& 2013 IEEE International Conference on Embedded and Ubiquitous Computing (HPCC\&EUC'13). 442-449. https://doi.org/10.1109/HPCC.and.EUC.2013.70

[20] Yuliang Li, Rui Miao, Mohammad Alizadeh, and Minlan Yu. 2019. DETER: Deterministic TCP Replay for Performance Diagnosis. In 16th USENIX Symposium on Networked Systems Design and Implementation (NSDI '19). USENIX Association, Boston, MA, 437-452. https://www. usenix.org/conference/nsdi19/presentation/li-yuliang

[21] Hongri Liu, Lun An, Jiawei Ren, and Bailing Wang. 2019. An Interactive Traffic Replay Method in a Scaled-Down Environment. IEEE Access 7 (2019), 149373-149386. https://doi.org/10.1109/ACCESS.2019.2947062

[22] Ali José Mashtizadeh, Tal Garfinkel, David Terei, David Mazieres, and Mendel Rosenblum. 2017. Towards Practical Default-On Multi-Core Record/Replay. In Proceedings of the Twenty-Second International Conference on Architectural Support for Programming Languages and Operating Systems (ASPLOS '17). Association for Computing Machinery, 693-708. https://doi.org/10.1145/3037697.3037751

[23] Ravi Netravali, Anirudh Sivaraman, Somak Das, Ameesh Goyal, Keith Winstein, James Mickens, and Hari Balakrishnan. 2015. Mahimahi: Accurate Record-and-Replay for HTTP. In Proceedings of the 2015 USENIX Conference on Usenix Annual Technical Conference (USENIX ATC '15). USENIX Association, 417-429. https://www.usenix.org/ conference/atc15/technical-session/presentation/netravali

[24] Soyeon Park, Yuanyuan Zhou, Weiwei Xiong, Zuoning Yin, Rini Kaushik, Kyu H. Lee, and Shan Lu. 2009. PRES: Probabilistic Replay with Execution Sketching on Multiprocessors. In Proceedings of the ACM SIGOPS 22nd Symposium on Operating Systems Principles (SOSP '09). Association for Computing Machinery, 177-192. https://doi.org/10.1145/1629575.1629593

[25] Shiru Ren, Chunqi Li, Le Tan, and Zhen Xiao. 2015. Samsara: Efficient Deterministic Replay with Hardware Virtualization Extensions. In Proceedings of the 6th Asia-Pacific Workshop on Systems (APSys '15). Association for Computing Machinery, 7. https://doi.org/10.1145/ 2797022.2797028

[26] Shiru Ren, Le Tan, Chunqi Li, Zhen Xiao, and Weijia Song. 2016. Samsara: Efficient Deterministic Replay in Multiprocessor Environments with Hardware Virtualization Extensions. In Proceedings of the 2016 USENIX Conference on Usenix Annual Technical Conference (USENIX ATC '16). USENIX Association, 551-564.

[27] Shiru Ren, Le Tan, Chunqi Li, Zhen Xiao, and Weijia Song. 2018. Leveraging Hardware-Assisted Virtualization for Deterministic Replay on 
Commodity Multi-Core Processors. IEEE Trans. Comput. 67, 1 (2018), 45-58. https://doi.org/10.1109/TC.2017.2727492

[28] R Senthilkumaran and Purushottam Kulkarni. 2014. Insight: A framework for application diagnosis using virtual machine record and replay. Department of Computer Science and Engineering Indian Institute of Technology, Tech. Rep. TR-CSE-2014-57 (2014).

[29] Yasser Shalabi, Mengjia Yan, Nima Honarmand, Ruby B. Lee, and Josep Torrellas. 2018. Record-Replay Architecture as a General Security Framework. In 2018 IEEE International Symposium on High Performance Computer Architecture (HPCA '18). IEEE, 180-193. https://doi.org/10. 1109/HPCA.2018.00025

[30] Gwendolyn Voskuilen, Faraz Ahmad, and T. N. Vijaykumar. 2010 Timetraveler: Exploiting Acyclic Races for Optimizing Memory Race Recording. In Proceedings of the 37th Annual International Symposium on Computer Architecture (ISCA '10). Association for Computing Machinery, 198-209. https://doi.org/10.1145/1815961.1815986

[31] Hao Wu, Hongri Liu, Bailing Wang, and Guodong Xin. 2017. Accurate traffic replay based on interactive sequence and timestamp. In 2017 IEEE 9th International Conference on Communication Software and Networks (ICCSN '17). 1107-1110. https://doi.org/10.1109/ICCSN.2017. 8230282
[32] Andreas Wundsam, Dan Levin, Srini Seetharaman, and Anja Feldmann. 2011. OFRewind: Enabling record and replay troubleshooting for networks. In Proceedings of the 2011 USENIX Conference on Usenix Annual Technical Conference (USENIX ATC '11). USENIX Association, 327-340. https://www.usenix.org/legacy/events/atc11/tech/final_files/ Wundsam.pdf

[33] Ruini Xue, Xuezheng Liu, Ming Wu, Zhenyu Guo, Wenguang Chen, Weimin Zheng, Zheng Zhang, and Geoffrey Voelker. 2009. MPIWiz: Subgroup Reproducible Replay of Mpi Applications. In Proceedings of the 14th ACM SIGPLAN Symposium on Principles and Practice of Parallel Programming (PPoPP '09). Association for Computing Machinery, 251260. https://doi.org/10.1145/1504176.1504213

[34] Lok-Kwong Yan, Manjukumar Jayachandra, Mu Zhang, and Heng Yin. 2012. V2E: Combining Hardware Virtualization and Softwareemulation for Transparent and Extensible Malware Analysis. In Proceedings of the 8th ACM SIGPLAN/SIGOPS Conference on Virtual Execution Environments (VEE '12). Association for Computing Machinery, 227-238. https://doi.org/10.1145/2151024.2151053

[35] Hyunmin Yoon, Shakaiba Majeed, and Minsoo Ryu. 2018. Exploring OS-Based Full-System Deterministic Replay (SAC '18). Association for Computing Machinery, 1077-1086. https://doi.org/10.1145/3167132. 3167247 